Il en résulte que la durée de la rotation varie d'une manière continue, de l'équateur à $\pm 45^{\circ}$ de latitule, d'environ 2 jours $\frac{1}{2}$. Cette loi de la variation de la vitesse angulaire en raison du carré du sinus de la latitude ne me parait pas être une simple formule empirique comme celles qui avaient été proposées jusque là pour représenter cette étonnante rotation, mais l'expression mathematique d'une loi bien singulière de la nature.

La figure du soleil étant sphérique et son axe conser. vant une direction constante dans l'espace, on voit que pour cet astre la rotation ordinaire n'est troublée que dans le sens des parallèles, j'en ai conclu que cette perturbation était due à lá présence de courants gazeux ascendants venant d'une profondeur de plus en plus grande à partir de l'équateur. Ces courants ne sont pas d'ailleurs une hypothèse gratuite. Les matières qui composent les petits amas incandescents de la photosphère étant à l'état solide, leur densité doit dépasser notablement celle du milieu gazeux où ils se for.ent. Ces matières solides doivent donc tomber vers le centre et produire ainsi des courants descendants lesquels doivent provoquer l'ascension d'autres courants verticaux qui aboutissent à la photosphère. Je pense que de là résulte l'alimentation et l'entretien de la photosphère par un jeu de combiuaisons chimiques qui s'opèrent à la surface et de décompositions chimiques qui s'opèrent dans les couches très profondes en vertu de leur excessive chaleur. Cette constitution interne explique l'enormité et la remarquable constance de la radiation solaire en faisant participer la masse entière à cette radiation.

Si lon tient compte des conditions thermodynamiques de ces courants ascendants, on voit qu'ils entretiennent dans les couches supérieures, au dessons de la photosphère et malgré la radiation interne de celle-ci, une sorte de chûte ou d'abaissement notable de température. De là résulte pour ces coucher déjà très denses malgré leur état de mobilité gazeuse, un pouvoir absorbant qui s'exerce sur la lumière émanée des régions centrales ou mème des régious diamétralement opposées, de la photosphère s'il en pouvait venir de là. Je crois avoir réussi à tenir compte, par cette simple remarque, d'une objection qui avait été faite à mon explication des taches par les Astronomes de Kew et par Mr. le Dr. Kirchhoff. Je m'explique en effet les taches comme de simples ouvertures daus la photosphère à travers lesquelles on aperçoit les couches gazeuses intérieures dont le faible pouvoir émissif doit contraster avec la vive radiation des matières solides de la photosphère.

Enfin la plupart des combinaisons chimiques qui s'accomplissent dans la photosphère peuvent l'opérer, grâce au jeu des courant verticaux, au moyen d'une quantitẻ restreipte d'oxygène accumulée dans les couches superficielles. La Terre nous offre quelque chose d'analogue, car le degré d'oxydation des matériaux qui en forment l'écorce solidifiée diminue avec la profondeur; l'intéricur est pent-être mème dépourvu d'oxygène à en juger par sa grande densité.

Cette théorie ou plutôt cette. ébauche me parait avoir l'avantage de relier à une simple conception mécanique les trois plus grands faits dont les astronomes et les physiciens se soient préoccupés, à savoir le mode de rotation, la grandeur et la longue durée d'une radiation constante depuis bien des siècles, et la noirceur des taches qui contraste avec le fonds éblouissant de la photosphère. Les personnes qui prendraient intérèt à ces idées trouveront de plus amples développemens daus les Comptes rendus de l'Acadenie, séance du 27 Juillet dernier.

$$
\text { Passy, le } 4 \text { Aout } 1868 . \quad \text { H. Faye. }
$$

\title{
Beobachtungen der Planeten (105) und (104) auf der Sternwarte zu Ann Arbor.
}

1 have observed the following places of a new planet discovered by me on the $16^{\text {th }}$ inst:

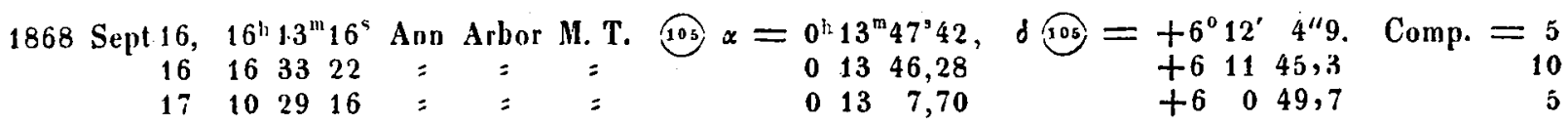

Planet 11.7 magnitude.

I add also further observations of Planet 104:

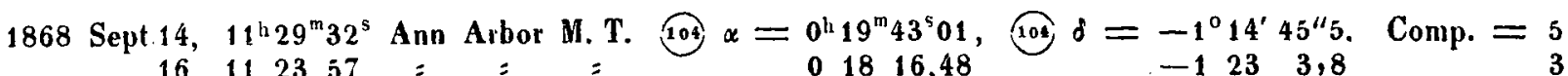
$16112357=\quad=\quad 01816,48$

Ann Arbor, 1868 September 18.

James C. Watson. 